\title{
LA EVALUACIÓN DE LOS PLANES DE CONVIVENCIA PARA LA REFLEXIÓN DE LA PRÁCTICA EDUCATIVA
}

\author{
THE EVALUATION OF COEXISTENCE PLANS \\ FOR THE REFLECTION OF EDUCATIONAL PRACTICE
}

\author{
Ana $\mathbf{M}^{\mathrm{a}}$ Moral Mora \\ Universidad de Valencia \\ ana.moral@uv.es \\ Amparo Pérez Carbonell \\ Universidad de Valencia \\ amparo.perez@uv.es \\ Inmaculada Chiva Sanchis \\ Universidad de Valencia \\ inmaculada.chiva@uv.es \\ Genoveva Ramos Santana \\ Universidad de Valencia \\ genoveva.ramos@uv.es
}

\section{RESUMEN}

Abordar la convivencia escolar ha sido objeto de numerosas investigaciones junto a diversas acciones dirigidas a las diferentes audiencias de la comunidad educativa. No obstante, se continúan produciendo situaciones conflictivas en los centros que, además de perpetuarse, engrandecen otras problemáticas que, de ser tratadas, a través de la prevención y la intervención adecuada, se gestionarían como naturales. Por tanto, es de especial relevancia plantearse el uso de un proceso de reflexión en torno a la convivencia en los centros en el que se incluya a toda la comunidad escolar: equipos directivos y educativos, personal de administración-servicios, gestores, familias y entidades sociales relacionadas con la escuela y, por supuesto, el alumnado. Presentamos los procesos de evaluación de planes de convivencia como los propicios para estos procesos de reflexión, resaltando aquellos puntos fuertes y débiles en los que hay que incidir. Con su uso por parte de toda la comunidad educativa, se aporta claridad en el camino del cambio necesario para que se pueda dar un ambiente de convivencia positivo que posibilite el desarrollo del alumnado. Por ello, se reclama junto a la necesaria participación de todas la personas involucradas en la vida de un centro, una mayor implicación y formación en elementos básicos relacionados con la evaluación que les permitan obtener información para profundizar en el descubrimiento de una educación que fomente la ansiada cohesión social.

Sabemos que la convivencia como marco del fomento de la cohesión social es un hecho que debe ser 
abordado en los centros escolares. Para ello, es prescriptivo establecer un plan de convivencia concreto en el que se incluya la participación de toda la comunidad escolar y en el que se planifique y se lleve a cabo también, por parte de dicha comunidad, su evaluación como parte del proceso de reflexión y mejora del mismo.

Palabras clave: evaluación, conflicto, conducta social, calidad de la educación, formación de profesores.

\begin{abstract}
Addressing school coexistence has been the subject of numerous investigations along with various actions directed at the different audiences of the educational community. However, conflicting situations continue to occur in centers that perpetuate and enhance other issues that, if addressed through prevention and appropriate intervention, would be managed as natural. Therefore, it is of special relevance to consider the use of a reflection process around the coexistence in the centers in which the entire school community is included: management and education teams, management-services personnel, managers, families and social entities related to the school and, of course, the students. We present the evaluation processes of coexistence plans as the propitious for these processes of reflection, highlighting those strengths and weaknesses in which we have to focus. With its use by the entire educational community, clarity is provided on the path of change necessary for an environment of positive coexistence that enables student development. For this reason, it is demanded the necessary participation of all the people involved in the life of a center, a greater involvement and training in basic elements related to the evaluation that allow them to obtain information to deepen the discovery of an education that foments the desired social cohesion.

We know that coexistence, as a framework for the promotion of social cohesion, is a fact that must be addressed in schools. For this, it is prescriptive to establish a concrete coexistence plan that includes the participation of the entire school community and in which it is planned and carried out by the community as well, as part of the reflection process and improvement thereof.
\end{abstract}

Key words: evaluation, conflict, social behaviour, quality of education, teacher education

\title{
INTRODUCCIÓN
}

La convivencia sigue siendo un tema de interés en la actualidad que motiva a la propuesta de iniciativas para su optimización desde las diferentes instituciones que conforman la sociedad. Concretamente en la escuela confluyen diversidad de agentes educadores, entre los que no siempre se dan relaciones fluidas siendo ellos mismos ejemplos incorrectos de lo que supone y debe ser un ambiente no conflictivo. Hablamos del profesorado, las familias, el personal administrativo y de servicios, son esos agentes que contribuyen a la educación del alumnado, protagonista y generalmente destinatario de los diversos procesos educativos que confluyen en los centros escolares.

Las familias, en ocasiones, con el ánimo protector y de cuidado a sus menores, a veces generan un ambiente caracterizado por la desconfianza, el reproche de lo realizado mostrándose así un binomio familia y escuela que es percibido por el profesorado del mismo como propósito de un control de lo que se realiza. Así como que las familias sienten que el profesorado juzga su educación familiar y los convierten en objeto de juicios y valoraciones, culpabilizándolas del comportamiento y falta de civismo de sus hijos-as. A ello se une la separación en los propios 
centros entre las funciones educativas y las de la administración- servicios y también de gestión, que provocan, en muchos casos, un funcionamiento en los centros inestable en cuanto a liderazgo de propuesta en el mismo para decidir, establecer y ejecutar un plan de convivencia anclado en la participación colectiva. Pequeña muestra, la señalada anteriormente, de la necesidad que existe de realizar una reflexión equilibrada, profunda y meditada por parte de toda la comunidad acerca de cuál es su situación, comportamiento, gestión y posición a tomar respecto a una situación de convivencia deseable (Otaduy, Quintana, Irigoyen y Zaitegui, 2006). Con esto ponemos de relieve el hecho de que, para que se produzca un cambio en los centros escolares en materia de convivencia, es necesaria la actuación integrada, es decir, la participación e implicación de toda la comunidad escolar a todos los niveles y actuaciones. Queda por tanto bien marcado que el diseño y elaboración de planes de convivencia en los centros es algo prescriptivo, que es de vital importancia hacerlo de una manera colegiada, planteando una situación de observación y reflexión tanto previa como de manera continuada a lo largo de su diseño y desarrollo, y exigiendo la participación de todos los miembros implicados en la educación. Estamos hablando de la necesidad no solo de ejecutar de manera coordinada y consensuada la prescripción que todo centro tiene de establecer un plan de convivencia, sino de que dicho proceso debe hacerse a través de un proceso de evaluación, que comienza en una reflexión previa, necesaria para que la intervención que se realice responda a los principios de calidad y necesidades de cada centro educativo. Así pues, proponemos centrarnos en dos ejes que consideramos se valoran, pero que en realidad no suelen realizarse, y que son la clave de nuestra propuesta:

1.- Incluir en los planes de convivencia un trabajo continuo de generación de espacios saludables de encuentro entre todos y cada uno de los implicados. Hemos de tener en cuenta que mejorando las relaciones entre los diferentes miembros de la comunidad escolar se propicia el diálogo y se deriva en un trabajo orientado hacia la cooperación y el consenso acerca de lo que debe ser la educación integral de las personas. Este hecho, contribuye a que se produzca una mayor cohesión social, tal y como indica uno de los objetivos de la Estrategia Europea para el 2020. En este punto, y de acuerdo con García Raga y López Martín (2010), entendemos que la convivencia escolar es un paso decisivo para alcanzar la convivencia social.

2.- La formación de los agentes educativos en la evaluación de los planes de convivencia proporciona los elementos clave en el proceso de optimización de la convivencia y de las relaciones entre los diferentes agentes educativos. Sin dicha evaluación, la planificación y la intervención se quedan cojas, imposibilitando el conocimiento de cómo nos encontramos, qué es lo que funciona y qué es lo que debe cambiarse o repensarse para el futuro. Como señalan Ortiz y Rivero (2007, p. 1):

Una teoría del cambio es como un mapa vial. Nos ayuda a planificar nuestro viaje desde el punto en el que estamos actualmente hasta dónde queremos llegar (...) nos ayuda a responder a la pregunta: ¿Cuál es el cambio que deseamos alcanzar y qué tiene que pasar para que este cambio se alcance?

Apostamos por alcanzar una convivencia positiva en los centros y lo que consideramos que debe pasar es que se produzca una reflexión documentada e informada para que se den los cambios necesarios orientados a la consecución de este objetivo. La evaluación es el proceso que puede 
facilitar y obtener las evidencias que permitan realizar el cambio que se necesita, es un ejercicio que proponemos como el motor del cambio para posibilitar una convivencia positiva en los centros educativos.

\section{OBJETIVO}

La pretensión de este trabajo es reflexionar acerca de la necesidad que existe de ser conscientes de que no solo hay que trabajar con el objetivo de formación para alumnado, formación para profesorado, formación para familias, sino que se debe apostar por una interformación - formación de toda la comunidad educativa - que de ejemplo en hechos. Esto exige tener muy clara la visión integral del estado de la convivencia del centro que no es posible sin la aportación de la percepción comentada y reflexionada por parte de todas las personas que viven el centro. Acción que proponemos se consigue usando la evaluación del plan de convivencia como estrategia dado que nos da a conocer de forma holística y concreta la situación de convivencia real.

La evaluación de los planes que se implementen pretende provocar la necesaria reflexión acerca de qué es lo que funciona y qué es lo que se debe optimizar, cuáles son las prácticas exitosas y las que deberían reconsiderarse en cuestiones que afectan a la convivencia, y, por tanto, al tipo de relaciones interpersonales que se están dando entre los miembros de la comunidad escolar.

\section{VIOLENCIA, CONFLICTO Y CONVIVENCIA}

Realmente la violencia es un fenómeno cuyas consecuencias influyen negativamente en el desarrollo personal y social de algunos alumnos y alumnas de primaria y secundaria (Defensor del Pueblo, 2007; Oñate y Piñuel, 2006). Además, la violencia transmite una serie de valores negativos que revierten en el uso de la fuerza, la agresión y la amenaza como modo de relación social dirigida al logro de los objetivos propios (Olweus, 2004). Estos valores se contradicen con el diálogo, la tolerancia, el respeto y la solidaridad, que son necesarios para poder vivir con los demás, para poder convivir.

Como señalan diferentes autores (Cascón 2000; Moral y Pérez Carbonell, 2010; Redorta, 2007), el conflicto es inherente a la persona y como tal, estamos abocados a vivir diversidad de situaciones conflictivas en nuestras múltiples interacciones diarias con aquellas personas con las que convivimos. Además, el conflicto es algo inherente a todas las organizaciones y, por tanto, también lo es en la escuela donde, como ocurre en el caso de las interacciones conflictivas también señaladas, según señalan Jares, (2008), Moral y Pérez Carbonell (2010) y Viñas (2004) es la forma en que se resuelve o gestiona lo que las diferencia.

El conflicto en nuestra sociedad es inevitable y forma parte de la vida de las personas como algo natural. Es un fenómeno activo y dinámico que se produce a causa de la divergencia de intereses o percepciones entre las personas. Como tal, puede ser asumido con una connotación positiva, en cuanto a ser vivido como una oportunidad para el crecimiento y el aprendizaje a lo largo de nuestras vidas o, con una connotación negativa, es decir, como algo destructivo e indeseable (Cornelius y Faire, 2003; Galtung, 2003; Vinyamata, 2005; Redorta, 2007). 
Existe una diversidad de conflictos que pueden suceder y afectar a las personas en el entorno escolar y van cambiando con el paso del tiempo. Por ejemplo, el uso de las nuevas tecnologías, por su rápida difusión e impacto multitudinario, generan un tipo de acoso que se va convirtiendo en otro de los problemas graves que afectan a la convivencia pacífica en los centros educativos, se trata del cyberbullying (Cerezo y Rubio, 2017; Gabarda, Orellana y Pérez Carbonell, 2017).

Para regular y contribuir a la promoción de la convivencia a través de la buena gestión de los conflictos en los centros escolares, la mayoría de comunidades autónomas crearon, sobre todo, entre los años 2005-2008, los “Observatorios de convivencia" a partir del propuesto por la administración central en el año 2004. Además, se consideraron algunas premisas para la elaboración de un plan de convivencia, que el Ministerio de Educación reguló ese mismo año 2004 (Cerezo y Rubio, 2017; Gómez Rivas, 2011; Otaduy et al., 2006; Ley Orgánica de Educación, 2006; Torrego, 2006). De este modo, y a partir de estos documentos, se fue forjando la normativa autonómica que impulsaba la creación de programas específicos que desarrollaran acciones concretas adaptadas a las características de cada uno de los centros educativos. En estos últimos años, se han ido actualizando las normativas que promueven la realización de dichos planes, estableciendo protocolos de actuación en caso de violencia escolar y siendo los reglamentos de régimen interno hojas de ruta en los que se establecen las normas de convivencia en los centros escolares.

Sin embargo, todavía queda mucho por hacer en cuanto al trabajo de la convivencia se refiere, puesto que, aunque haya una normativa de base, se muestra muy poco específica en cuanto a la puesta en marcha de su operatividad a través de planes y presenta algunas contradicciones respecto al carácter de las medidas propuestas. Así, Cerezo y Rubio (2017) en su estudio, comparan la normativa acerca de convivencia que promulgan ocho comunidades autónomas españolas. Reflejan que más del 80\% de las medidas que se impulsan son de carácter correctivo o disciplinario, siendo las de carácter educativo totalmente inexistentes, aunque en dicha normativa se afirma que sí tienen carácter educativo. En cambio, en la revisión realizada por Pérez Carbonell, Ramos y Serrano (2017) se concluye que cuando se habla con el profesorado éste señala que conoce y desarrolla más acciones de prevención primaria y secundaria sobre el acoso escolar, de enfoque más educativo, que de prevención terciaria con un claro enfoque más punitivo. Sin embargo, estas mismas autoras, señalan que casi el $40 \%$ de las comunidades autónomas, no contemplan medidas de protección o apoyo a las víctimas de las agresiones lo que se traduce en una regulación autonómica insuficiente y contradictoria, que, alentando a la creación de medidas de naturaleza educativa para la promoción de la convivencia en los centros, tan sólo propone protocolos dirigidos a acciones de tipo disciplinario y fundamentalmente orientados al trabajo con las personas agresoras.

Ya hace unos años que se dejó clara la necesidad de generar intervenciones contando con todos los miembros de la comunidad escolar, alentar la creación y desarrollo de las comisiones de convivencia como órganos colegiados que actúan por delegación de los consejos escolares y que se componen por representantes de cada colectivo de la comunidad escolar (Torrego, 2006). Diversos estudios muestran que las iniciativas cuyo objetivo es mejorar la convivencia en los centros escolares y que cuentan con la participación de todos sus miembros o, al menos gran parte, consiguen que se produzca un cambio considerable en el ambiente del centro; aunque también se dan experiencias en las que en algunos de los centros en dónde se implementan acciones dirigidas a algunos de los 
colectivos concretos (profesorado y/o alumnado), se producen resultados que afectan positivamente a otros miembros y se da una mejora en las relaciones interpersonales entre profesorado, familias y alumnado (Boqué y García Raga, 2010; Cerezo y Rubio, 2017; De Prada, 2014; Moral, Ramos y Chiva, 2014).

En la actualidad, se han difundido unas guías promovidas por la administración central, con el objetivo de que se produzca una implantación definitiva de los planes de convivencia en los centros, se conoce como Plan estratégico de convivencia (2016). Dicho Plan, responde a la necesidad suscrita por diversos estudios (Serrano e Iborra, 2005; Díaz Aguado, Martínez y Martín, 2013), además de las exigencias a las que el Estado Español se ve comprometido y que promulgan las Naciones Unidas y la Unión Europea en materia de la protección de los derechos de los niños y niñas, además de la búsqueda de la cohesión social y una mayor participación ciudadana. Los objetivos fundamentales del Plan estratégico son los siguientes (Plan estratégico de convivencia, 2016):

- Asegurar la inclusión de todo el alumnado en los centros educativos.

- Ampliar y fortalecer la participación de las familias y la comunidad en la escuela.

- Desarrollar procesos integrales de educación de los sentimientos y las emociones de manera transversal a la adquisición de aprendizajes académicos.

- Asegurar la incorporación de las temáticas relacionadas con la convivencia escolar en la formación inicial y permanente del profesorado.

- Asegurar el trabajo de prevención de la violencia desde la primera infancia.

- Desarrollar actuaciones de socialización preventiva de la violencia de género.

- Garantizar que la mejora de la convivencia se traslade a los espacios de socialización asociados a las TIC.

Entre las principales líneas de actuación destacan la que ocupa el cuarto lugar, la importancia de la formación del profesorado. Se destaca su papel como modelo y promotor de valores positivos en el alumnado, así como su implicación en la consecución del resto de los ejes que se nombran a continuación:

1. Observación y seguimiento de la convivencia en los centros educativos.

2. Desarrollo de políticas educativas para la mejora de la convivencia.

3. Incorporación de Actuaciones Educativas de Éxito y prácticas basadas en criterios científicos para la mejora de la convivencia escolar.

4. Formación del profesorado y otros agentes de la comunidad educativa.

5. Coordinación y cooperación entre administraciones, entidades e instituciones.

6. Prevención y control de incidentes violentos en los centros educativos y apoyo a las víctimas de violencia y acoso. 
7. Comunicación, intercambio y difusión de información y conocimiento sobre el impacto de la convivencia escolar en la educación.

8. Investigación educativa y social en convivencia escolar.

Pensemos y revisemos, la mayoría de objetivos y/o ejes que se demandan: "asegurar inclusión (...)", "ampliar y fortalecer (...)", "desarrollar actuaciones (...)", "observación y seguimiento (...)", "comunicación, intercambio y difusión (...)", son actuaciones que, necesariamente, exigen un proceso de evaluación por parte de los agentes implicados.

\section{LA NECESIDAD DE EVALUAR LOS PLANES DE CONVIVENCIA EN LOS CENTROS ESCOLARES}

Si partimos de la base de que la evaluación educativa es un proceso complejo, dinámico, continuo y sistemático que puede actuar como un potente instrumento de cambio de la realidad educativa (Lukas y Santiago, 2004), necesariamente se tratará de un instrumento a utilizar a la hora de poner en marcha o valorar cualquier plan de convivencia en los centros educativos. Siguiendo a Pérez Juste (2000), desde una perspectiva integradora, se tratará de llevar a cabo un proceso sistemático, diseñado intencional y técnicamente, de recogida de información rigurosa -valiosa, válida y fiable- orientado a valorar la calidad y los logros de un programa, como base para la posterior toma de decisiones de mejora, tanto del programa como del personal implicado y, de modo indirecto, del cuerpo social en que se encuentra inmerso.

De acuerdo con este concepto, la evaluación debe dejar su lastre de "control" y pasa a ser utilizada para "mejorar", y ello es así porque gracias a ella podemos conocer realmente lo que estamos haciendo. Nos permite planificar y contrastar, explicar cómo funciona un programa respondiendo a qué queremos hacer, dónde queremos llegar y qué cambios o continuidad a mantener para alcanzar lo que deseamos. Nos aporta, en definitiva, un conocimiento coherente de lo realizado y del resultado obtenido.

La evaluación de programas que previenen la violencia y promocionan la convivencia en los centros escolares, es un elemento que se considera relevante y se planifica en casi todos los programas que tratan de prevenir la violencia en las aulas. Sin embargo, en pocas ocasiones se lleva a cabo (Torrego y Galán, 2008), siendo como ya señalaba Smith (2003), un tema pendiente en las iniciativas y actuaciones realizadas y más, sobre todo, en un Centro Escolar. Es difícil encontrar, a través de la búsqueda documental, el desarrollo de evaluaciones de dichos programas, a pesar de ser un aspecto que se recomienda y se entiende como fundamental, pero que por unos motivos u otros no acaba de desarrollarse (Perochena, Olmos, Herrera y Rodríguez Conde, 2009). Una vez hecha la planificación y el diseño de cualquiera de los programas de prevención de la violencia escolar orientado al fomento de la convivencia siempre surgen interrogantes, cómo: ¿qué es lo que puede asegurar el funcionamiento del programa?, ¿cómo comprobar que realmente se produce una mejora de la convivencia en el centro? y, si no se ha producido mejora, ¿cómo podemos incluir elementos que optimicen el programa? La necesidad de evaluar los planes y programas de convivencia que han sido diseñados e implementados en los centros educativos se justifica en cuanto que supone: 
- El análisis inicial de las necesidades y recursos de cada centro, anterior a la implementación del programa,

- Un modo de obtención de conocimiento acerca del funcionamiento del programa mientras se realiza y una vez implementado, y, sobre todo,

- Una oportunidad para la reflexión individual y colectiva de lo realizado que conduce siempre a la mejora.

El uso de la evaluación de los planes de la convivencia en los centros educativos supone el motor de cambio necesario orientado a evidenciar aquellas prácticas educativas que mejoran y optimizan las relaciones personales generando un clima positivo de convivencia. Eso sí, somos también conscientes de que no toda evaluación sirve.

\section{ELEMENTOS PARA LA EVALUACIÓN DE LOS PLANES DE CONVIVENCIA ESCOLAR}

Entendemos que todo proceso de evaluación debe tener un modelo de guía o referente, pues como señala Municio "un modelo de evaluación es como un mapa de carreteras que permite localizar el lugar donde se está...El modelo proporciona la ruta o rutas que permiten pasar de donde estamos a donde queremos estar" (Pérez Juste, López Rupérez, Peralta, y Municio Fernández, 2000, p. 175). Sin embargo, no podemos olvidar que los modelos son referentes globales y, por tanto, deben ser adaptados a los casos concretos. Motivo por el que el responsable de la evaluación de los planes de convivencia no se puede adherir a modelos utilizados en otras propuestas dado la necesidad de su contextualización, contenido y pretensiones, aunque, si es cierto que debe compartir elementos de otros modelos evaluativos. En esta línea, los diferentes elementos que se deben atender y que se marcan en otras evaluaciones (De Miguel, 2000; Fernández-Ballesteros, 1995; Pérez Carbonell, 1998; Pérez Juste, 2000; Smith, 2003) son los que aparecen a continuación (ver Tabla 1): 


\begin{tabular}{|c|c|c|}
\hline COMPONENTE & PREGUNTA & RESPUESTA \\
\hline Objeto & $\begin{array}{l}\text { ¿Qué se } \\
\text { evalúa? }\end{array}$ & $\begin{array}{l}\text { Va a ser evaluado: "La comunidad educativa en diseño, implantación y gestión del } \\
\text { Plan de convivencia" }\end{array}$ \\
\hline Finalidad & $\begin{array}{l}\text { ¿Para qué se } \\
\text { evalúa? }\end{array}$ & $\begin{array}{l}\text { Formativa: MEJORA } \\
\text { Sumativa: RENDICIÓN DE CUENTAS } \\
\text { En este caso en que se evalúa la situación de convivencia y la gestión que se realiza } \\
\text { a nivel de centro e implicados de la misma, es más relevante la evaluación formativa, } \\
\text { centrada en procesos más que en resultados, aunque la sumativa da información } \\
\text { acerca de cuántos casos se han resuelto satisfactoriamente y es, por ejemplo, un } \\
\text { indicador que puede también ser relevante si estamos pensando en buscar equilibrio } \\
\text { entre recursos y alcance. }\end{array}$ \\
\hline $\begin{array}{l}\text { Audiencias/ } \\
\text { Fuentes }\end{array}$ & $\begin{array}{c}\text { ¿Quién está } \\
\text { implicado? } \\
\text { ¿Qué } \\
\text { información } \\
\text { pueden } \\
\text { aportar? }\end{array}$ & $\begin{array}{l}\text { - Estudiantes } \\
\text { - Docentes } \\
\text { - Personal no docente } \\
\text { - Familias } \\
\text { - Entidades colaboradoras } \\
\text { Hablamos de quién o quienes pueden participar en el proceso evaluativo. Tal y } \\
\text { como lo planteamos sería toda la comunidad comentada. A cada una le pediríamos } \\
\text { la información que se precisará atendiendo al planteamiento realizado en el Plan de } \\
\text { Convivencia, aunque sería aconsejable buscar alguna información en todas que nos } \\
\text { permitiera contrastar la percepción ante el mismo hecho de cada audiencia. También } \\
\text { hay que atender a quién/quiénes van a realizar la evaluación (si lo hace quien lo ha } \\
\text { diseñado y/o implementado, será señalado como más subjetivo y se podrá asumir su } \\
\text { información como menos convincente. Si la realizan personas externas al mismo se } \\
\text { habla de mayor objetividad, aunque se da una menor comprensión de lo ocurrido). } \\
\text { En nuestro caso optaríamos siempre por la realización de la evaluación de audiencias } \\
\text { internas al centro. }\end{array}$ \\
\hline Instrumentos & $\begin{array}{l}\text { ¿Cómo se } \\
\text { recoge la } \\
\text { información? }\end{array}$ & $\begin{array}{l}\text { - Técnicas evaluativas } \\
\text { - Instrumentos de medida } \\
\text { Selección de instrumentos y técnicas de recogida de información de las audiencias } \\
\text { implicadas en el proceso evaluativo }\end{array}$ \\
\hline Momentos & $\begin{array}{c}\text { ¿Cuándo } \\
\text { se recoge la } \\
\text { información? }\end{array}$ & $\begin{array}{l}\text { - Previo } \\
\text { - Inicio } \\
\text { - Final } \\
\text { - Posterior } \\
\text { Determinar los momentos en los que se lleva a cabo la obtención de información }\end{array}$ \\
\hline $\begin{array}{l}\text { Análisis de la } \\
\text { información }\end{array}$ & $\begin{array}{l}\text { ¿Cómo se } \\
\text { analiza y } \\
\text { sintetiza? }\end{array}$ & $\begin{array}{l}\text { - Análisis estadístico (análisis descriptivo-inferencial) } \\
\text { - Análisis de contenido } \\
\text { Tipo de análisis que se van a utilizar para el tratamiento de la información recogida. } \\
\text { Básicamente se escogen dependiendo de si la información recogida es cualitativa } \\
\text { o cuantitativa. En el primer caso, por ejemplo, se llevaría a cabo un análisis de } \\
\text { contenido y en el segundo un análisis estadístico que puede ser tan básico o complejo } \\
\text { como requiera la situación. }\end{array}$ \\
\hline $\begin{array}{l}\text { Difusión de la } \\
\text { información }\end{array}$ & $\begin{array}{c}\text { ¿Cómo se } \\
\text { difunde la } \\
\text { información? }\end{array}$ & $\begin{array}{l}\text { - Informes } \\
\text { La difusión de la información ha de realizarse de acuerdo a unos canales que se } \\
\text { consideren válidos (escrito, oral, etc.). La máxima en este caso es que sea cual sea el } \\
\text { modo de comunicación, ésta se adapte a la comprensión de las audiencias a las que } \\
\text { vaya destinada. }\end{array}$ \\
\hline
\end{tabular}

Tabla 1. Síntesis de los elementos básicos para la evaluación de Planes de convivencia escolar. Fuente: Adaptado de Moral y Pérez Carbonell (2010) 
Estas son algunas de las cuestiones que deberíamos poder responder respecto a la evaluación de programas fundamentados en la promoción de la convivencia escolar. Si nos damos respuesta a estas preguntas conseguimos una revisión y un análisis de la realidad educativa de los centros y para hacerlo se deben buscar o, por la especificidad del programa y contexto, elaborar instrumentos exhaustivos o generar dinámicas de recogida de información ricas en comprensión de lo ocurrido. No podemos desde la teoría presentar un instrumento ideal y adecuado a cada programa, a cada centro, a cada comunidad educativa, además no es ese nuestro objetivo. Lo que pretendemos es transmitir la necesidad, el convencimiento de que lo que si debe permitir toda recogida de información es una "panorámica en movimiento", apostamos por un planteamiento global como el del Index for Inclusion (Booth y Ainscow, 2000). Hablamos de un instrumento que aporta una serie de materiales que permiten desarrollar procesos de innovación en los centros escolares que contribuyan al desarrollo de valores compartidos entre todas las personas que conforman la comunidad educativa, a la mejora de la participación fundamental y necesaria de todo el alumnado, además de la mejora del aprendizaje en aquello en lo que se trabaja dado que propicia la sintonía de la colaboración (Sandoval, López, Miquel, Durán, Giné y Echeita, 2013). Se trata de un instrumento que propone el análisis de la situación educativa de cada centro atendiendo a una serie de fases en las que se aportan unos indicadores concretos para el trabajo de cada una de ellas. Su implementación, implica el compromiso por parte de un grupo de miembros de la comunidad escolar a realizar un análisis de la situación de convivencia de cada centro a lo largo de un periodo de tiempo que puede durar varios cursos académicos.

\section{CONCLUSIÓNY DISCUSIÓN}

La generación de una cultura de evaluación en los Planes que se realizan en los centros creemos es la clave para generar un cambio efectivo.

De modo que, siguiendo las pautas de proceso del Index, planteamos:

1. Constituir un grupo con personas de todos los colectivos que realizan un estudio de necesidades de convivencia en el centro. Grupo convencido, motor del proceso bien por conocimiento del tema desde la teoría o por convencimiento tras la experiencia en el centro. Grupo motivador. Identifica la realidad, indaga sobre buenas prácticas, programas formativos. Es un grupo detector de conocer qué pasa, cómo pasa, qué se necesita para que lo que pase sea una mejora en convivencia y conflicto escolar.

2. Grupo que comienza procesos de exploración del grado de conocimiento que la comunidad educativa tiene sobre la convivencia que se da en el centro y que propicia una valoración conjunta de la situación real del centro desde todas las miradas.

3. Grupo que trabaja la sensibilización de toda la comunidad educativa en la importancia de trabajar en convivencia en el centro y que planifica una guía de trabajo poniendo de relieve las prioridades detectadas. Para así poder elegir o elaborar un programa que se centre en dar respuesta a dónde estamos y a dónde queremos llegar, porque así se realizará una definición del cambio que deseamos alcanzar. Situación presente y situación futura posible. 
4. Integración de toda la comunidad educativa en esta perspectiva como grupo que de manera conjunta quiere construir un espacio de convivencia y no conflicto que llegue a ser un ejemplo por y para todos y todas. Hay que generar un sentimiento de pertenencia al centro.

5. Elección o elaboración de un programa en el que participa el Centro de manera global en el que se queda marcada toda acción prioritaria y en el que aparece cómo se deben registrar los progresos de todos los cambios que se dan a través de la revisión de indicadores que se han marcado.

6. El grupo motor recoge y transcribe el resultado dando a conocer el alcance de las decisiones adoptadas y los cambios alcanzados.

La situación de violencia estructural que se vive en la sociedad actual, hace que los organismos internacionales promuevan medidas dirigidas a la inclusión de todas las personas en la sociedad, así como a la mejora de la cohesión social y la ciudadanía participativa. Como reflejo de lo que pasa en la sociedad, las instituciones educativas también se ven afectadas por fenómenos como el bullying y cyberbullying. Para ello, y desde hace un par de décadas se llevan planteando por parte de la administración la regulación de normativas y de los Planes de convivencia y mecanismos que contribuyan a una convivencia positiva en los centros. Sin embargo, todavía es necesario insistir en el apoyo de este tipo de normativas, puesto que el desarrollo de la convivencia es fundamental para que se produzca un cambio social necesario para potenciar la cohesión y la participación ciudadana. Como hemos ido planteando desde el inicio, creemos que generalmente las iniciativas hasta ahora emprendidas no han tenido los resultados esperados dado que no han partido del uso de una evaluación de partida, de un proceso de reflexión conjunta y continuada dentro de cada centro. La apuesta es además que ese proceso se inicie desde el comienzo y que además se cree o al menos potencie la necesidad de implicación y reflexión por todos y todas.

Entre las líneas de actuación por las que se apuesta, el plan estratégico para el fomento de la convivencia en los centros escolares, destacan la formación del profesorado y la importancia de la práctica docente para la consecución del cambio educativo necesario orientado a la promoción de valores positivos en el alumnado que potencien actitudes facilitadoras de una convivencia óptima. La implementación de iniciativas orientadas a la convivencia escolar debe contar con la implicación de toda la comunidad educativa para que realmente se produzca un cambio. Por tanto, se hace prioritario replantearse determinadas prácticas educativas a través de un análisis de la situación de convivencia de cada centro educativo.

Establecer espacios de diálogo se hace imprescindible para reunir a los diferentes colectivos implicados en la comunidad escolar y diagnosticar la realidad, exponer ideas desde la propia experiencia que, compartida pueda aportar soluciones a las diversas situaciones que tengan lugar en los centros. Como se ha comentado, la evaluación propicia los elementos y la guía para comenzar y desarrollar un proceso de reflexión orientado a la mejora y al cambio en las instituciones escolares. Existen instrumentos, que se han implementado, que se pueden adaptar a cada contexto o que se pueden diseñar, que nos permitan conocer qué está pasando en la realidad educativa. Para ello, se hace necesario un compromiso con la formación y la preparación de profesorado, familias y demás agentes educativos, generando estrategias para ejercer dicha evaluación o siguiendo con los procesos 
que ya se están llevando a cabo en los centros escolares. Los resultados aportarán información acerca de por un lado, cuál es la situación de partida de un centro y poder diseñar así un plan de convivencia adaptado y particular de cada centro educativo, por otro, una vez puesto en marcha el plan de convivencia reflexionar de manera continua sobre qué es lo que debería cambiar, qué es lo que funciona para que se pueda dar un clima positivo favorable a la convivencia entre todos y todas.

En definitiva, para que se produzca un cambio orientado a la mejora de la convivencia en los centros escolares, se debe tener en cuenta lo siguiente:

- Legislación y apoyo normativo de base por parte de la administración.

- Formación del profesorado por su papel como modelo educativo para el alumnado y aspecto fundamental en el desarrollo de las etapas dirigidas a la intervención para la mejora de la convivencia escolar.

- Implicación de toda la comunidad escolar para que se produzca un cambio real y consensuado a través del diálogo y la participación de todos los agentes educativos implicados en el proceso de enseñanza aprendizaje.

- Análisis de la situación de convivencia para la reflexión orientada al cambio en las prácticas educativas de cada centro educativo.

- Por lo que se hace necesaria una evaluación orientada al cambio de actitudes y valores, para llevar a cabo esos procesos de reflexión en los que debe participar toda la comunidad escolar desarrollando iniciativas y modelos educativos en los que la convivencia positiva en los centros sea prioritaria.

Entendemos que el camino no es fácil, sin embargo, estamos convencidas de que la única manera de que se produzca un cambio, es trabajar en ello y analizar cuáles son los modelos de relación que estamos enseñando como educadores ya sea en las aulas, en las familias, en definitiva, en la sociedad. Para poder modificar aquello que no contribuye al respeto, la tolerancia de las otras personas y que dificulta la convivencia en los centros, en las familias y en la sociedad. Potenciando de este modo aquellas prácticas y modelos que favorezcan relaciones de colaboración en la comunidad escolar.

\section{REFERENCIAS BIBLIOGRÁFICAS}

Booth, T. y Ainscow, M. (2000). Index for Inclusion: developing learning and participation in schools. Bristol: CSIE.

Boqué, M. C. y García Raga, L. (2010). Evaluación diferida de la formación del profesorado en convivencia y mediación. Revista Electrónica Interuniversitaria de Formación del Profesorado, 13(3), 87-94. Recuperado de: http://www.redalyc.org/articulo.oa?id=217015214008

Cascón, P. (2000). Educar en y para el conflicto en los centros. Cuadernos de Pedagogía, 287, 61-66.

Cerezo, F. y Rubio, F. J. (2017). Medidas relativas al acoso escolar y ciberacoso en la normativa autonómica española. Un estudio comparativo. Revista Electrónica Interuniversitaria De Formación Del Profesorado, 20(1), 113-126. DOI: https://doi.org/10.6018/reifop/20.1.253391 
Cornelius, H. y Faire, S. (2003) (5 Ed.). Tú ganas yo gano. Cómo resolver conflictos creativamente. Madrid, España: Editorial Gaia.

De Miguel, M. (2000). La evaluación de programas sociales. Fundamentos y enfoques teóricos. Revista de Investigación Educativa, 18 (2), 289-318.

De Prada, J. (2014). Modelo para la incorporación de un sistema de Mediación Escolar en un centro educativo de Educación Secundaria Obligatoria. Innovación Educativa, 24, 35-47. DOI: https://doi.org/10.15304/ie.24.1928

Defensor del Pueblo (2007). Violencia escolar: el maltrato entre iguales en la Educación Secundaria Obligatoria 1999-2006. Nuevo estudio y actualización del informe 2000. Recuperado de https://www.defensordelpueblo.es/informe-monografico/violencia-escolar-el-maltrato-entre-iguales-en-la-educacion-secundaria-obligatoria-1999-2006-nuevo-estudio-y-actualizacion-del-informe-2000-2007/

Díaz-Aguado, M. J., Martínez, R. y Martín, J. (2013). El acoso entre adolescentes en España. Prevalencia, papeles adoptados por todo el grupo y características a las que atribuyen la victimización. Revista de Educación, 362, 348-379.

Fernández-Ballesteros, R. (Ed.) (1995). Evaluación de Programas. Una Guía Práctica en Ámbitos Sociales, Educativos y de Salud. Madrid, España: Editorial Síntesis.

Gabarda, S., Orellana Alonso, N. y Pérez Carbonell, A. (2017). La comunicación adolescente en el mundo virtual: una experiencia de investigación educativa. Revista de Investigación Educativa, 35(1), 251-267. DOI: https://doi.org/10.6018/rie.35.1.251171

Galtung, J. (2003). Paz por medios pacíficos. Paz y conflicto, desarrollo y civilización. GernikaGogoratuz, España: Colección Red Gernika.

García Raga, L. y López Martín, R. (2010). La convivencia escolar. Una mirada pedagógica, política y prospectiva. Valencia, España: Universitat de València.

Gómez Rivas, F. A. (2011). La normativa sobre convivencia escolar en España. Revista Amazónica, 6 (1), 149-185. Recuperado de: http://dialnet.unirioja.es/servlet/oaiart?codigo=3915955

Jares, X. R. (2008). Pedagogía de la convivencia (1. reimpr. ed.). Barcelona, España: Graó.

Ley Orgánica de Educación (LOE) (2006). 2/2006, de 3 de mayo. BOE de 4 de mayo.

Lukas, J. F. y Santiago, K. (2004). Evaluación Educativa. Madrid, España: Alianza Editorial.

Ministerio de Educación, Cultura y Deporte (2016). Plan estratégico de convivencia escolar 20162020. Recuperado de https://www.mecd.gob.es/educacion-mecd/dms/mecd/educacion-mecd/ mc/convivencia-escolar/plan-de-convivencia/Plan-estrategico-Convivencia-Escolar.pdf

Moral, A. Ma y Pérez Carbonell, Mª D. (2010). La evaluación del Programa de Prevención de la Violencia Estructural en la Familia y en los Centros Escolares. Revista Española de Orientación y Psicopedagogía, 21(1), 25-36. DOI: https://doi.org/10.5944/reop.vol.21.num.1.2010.11496

Moral, A. Ma , Ramos, G. y Chiva, I. (2014). Los programas de convivencia escolar y los modelos de percepción subyacentes de sus implicados. Intersticios: Revista sociológica de pensamiento crítico, 8, 1, 133-147.

Olweus, D. (2004) (2 ${ }^{\mathrm{a}}$ Ed.). Conductas de acoso y amenaza entre escolares. Madrid, España: Morata.

Oñate, A. y Piñuel, I. (2006). Estudio Cisneros X: Violencia y acoso escolar en España. Madrid, España: Instituto de Innovación Educativa y Desarrollo Directivo. 
Ortiz, A. y Rivero, G. (2007). Desmitificando la Teoría del Cambio. PACT ediciones. Recuperado de: http://www.rootchange.org/about us/resources/publications/DemistificandolaTeoriadeCambio.pdf

Otaduy, M., Quintana, G., Irigoyen, M. y Zaitegi, N. (2006). Guía para la elaboración del Plan de Convivencia Anual (PCA). Organización y gestión educativa: Revista del Fórum Europeo de Administradores de la Educación (OGE), 14 (4), 45.

Pérez Carbonell, A. (1998). Metodología de la Evaluación de Programas: Evaluación de Programas de Postgrado en la Universitat de Valencia. Tesis Doctoral. Valencia, España: Servei de Publicacions de la Universitat de Valencia.

Pérez Carbonell, A., Ramos Santana, G. y Serrano Sobrino, M. (2017). Acciones de prevención en acoso escolar utilizadas por docentes de Educación Secundaria Obligatoria. Contextos Educativos. Revista de Educación, 2, 163-179, DOI: https://doi.org/10.18172/con.2954

Pérez Juste, R. (2000). La evaluación de programas educativos: conceptos básicos, planteamientos generales y problemática. Revista de Investigación Educativa, 18, 2, 261-287.

Pérez Juste, R., López Rupérez, F., Peralta, Ma D. y Municio Fernández, P. (2000). Hacia una educación de calidad: gestión, instrumentos y evaluación. Madrid, España: Narcea.

Perochena, P., Olmos, S, Herrera, M. E. y Rodríguez Conde, M. J. (2009). Validación de un programa experimental de desarrollo de convivencia escolar en ESO, basado en el uso de blogs. Actas XIV Congreso Nacional De Modelos De Investigación Educativa "Educación, Investigación Y Desarrollo Social”.

Redorta, J. (2007). Cómo analizar los conflictos. La tipología de conflictos como herramienta de mediación. Barcelona, España: Paidós.

Sandoval M., López, M. L., Miquel, E., Durán, D., Giné, C. y Echeita, G. (2013). Index for Inclusion: Una guía para la evaluación y mejora de la educación inclusiva. Contextos Educativos: Revista de Educación, (5), 227-238. DOI: https://doi.org/10.18172/con.514

Serrano, A. e Iborra, I. (2005). Informe Violencia entre compañeros en la escuela. Valencia, España: Centro Reina Sofía para el estudio de la violencia.

Smith, P. (2003). Violence in schools. The response in Europe. London, England: Routledge Falmer. DOI: https://doi.org/10.1891/vivi.2003.18.1.55

Torrego, J. C. (Coord.) (2006). Modelo integrado de mejora de la convivencia. Estrategias de mediación y tratamiento de conflictos. Barcelona, España: Graó.

Torrego, J. C. (2008) (Coord.). El plan de convivencia. Fundamentos y recursos para su elaboración $y$ desarrollo. Madrid, España: Editorial Alianza.

Torrego, J. C. y Galán, A. (2008). Investigación evaluativa sobre el programa de mediación de conflictos en centros escolares. Revista de Educación, 347, 369-394.

Viana-Orta, Ma I. (2014). La mediación escolar en los planes y programas institucionales de convivencia en España/ School mediation in institutional plans and programmes of school coexistence in Spain. Revista Complutense De Educación, 25(2), 271-291. DOI: https://doi. org/10.5209/rev RCED.2014.v25.n2.41458

Vinyamata, E. (2005) (2a Ed.). Conflictología. Curso de resolución de conflictos. Barcelona, España: Ariel.

Viñas, J. (2004). Conflictos en los centros educativos: cultura organizativa y mediación para la convivencia. Barcelona, España: Graó. 